\title{
Influence of core and hexapod geometry, and local reinforcement on the performance of ultra-lightweight ULE mirror
}

William R. Arnold ${ }^{a}$, H. Philip Stahla, ${ }^{a}$ NASA Marshall Space Flight Center, Redstone Arsenal, Huntsville, AL, USA 35812

\begin{abstract}
The Habitable Exoplanet Observatory (HabEx) mission has unique optical performance requirements which drive the mirror design process beyond the traditional criteria. While mass and stiffness are still important, the response to inertia loading (expressed in terms of Zernike coefficients) to omni-directional excitation dominates the effort. While a Zerodur mirror is the current baseline, as mass budgets change, a ULE design is being studied as a potential alternative. This trade study looked at over 264 design variations using the Arnold Mirror Modeler and ANSYS $^{\odot}$ to investigate the influence of various design elements, including: substrate thickness, core cell size, hexapod geometry and local reinforcement. Design 'goodness' was evaluated based on the mirror's inertial deformation response to omni-directional input. This response was calculated via RSSing Zernike polynomial responses to (XYZ) accelerations.
\end{abstract}

Keywords: HabEx, primary mirror design, trade studies, ULE alternatives

\section{INTRODUCTION}

The HabEx Architecture-A telescope requires a 4-meter off-axis circular-aperture monolithic primary mirror. An openback Zerodur mirror is the baseline, with a closed-back ULE mirror as an alternative. This paper is one in a series of design studies supporting the project. Previous papers ${ }^{1,2}$ covered the Zerodur option extensively and limited ULE cases as well as discussing the overall scope of the HabEx work at MSFC. This paper expands the ULE design parameters with an emphasis on the impact of suspension system geometry and local reinforcement. The HabEx mission and the unique requirements of the coronagraph control the output format of this study. For HabEx, inertial deformation (response to inertial loads) is an important performance parameter both as it relates to manufacturing for diffraction limited performance and to ultra-stable on-orbit wavefront performance required by coronagraph.

The ranges of all the parameters used in this study are set by published industrial capabilities. The actual mirror manufacturer may or may not be the raw material supplier. A total of 264 separate models were created and run in period of two weeks using the AMM (Arnold Mirror Modeler) and ANSYS.

\subsection{Purpose of HabEx}

The basic goals of the HabEx Program are explained in Figure 1.

EXPLORING PLANETARY SYSTEMS AROUND NEARBY SUNLIKE STARS

AND ENABLING OBSERVATORY SCIENCE FROM THE UV THROUGH NEAR-IR

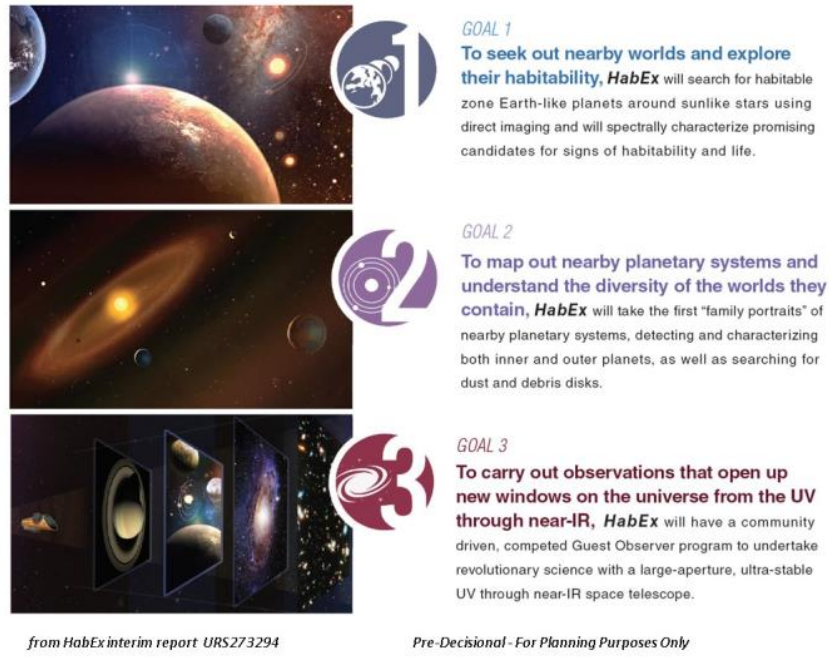

Figure 1: Basic goals of HabEx program 


\subsection{Baseline HabEx Architecture A Concept}

The HabEx Science and Technology Definition Team (STDT) chose the following parameters for Architecture-A (Figure 2). The telescope would have a 4-meter aperture, with a 72-meter external Starshade occulter flying in formation with the satellite. Four instruments would be included on the satellite; a coronagraph instrument for Exoplanet Imaging, a Starshade Instrument for Exoplanet Imaging, a UV-NIR Imaging Multi-object Slit Spectrograph for general observatory science and a High-Resolution UV Spectrograph for general observatory science.

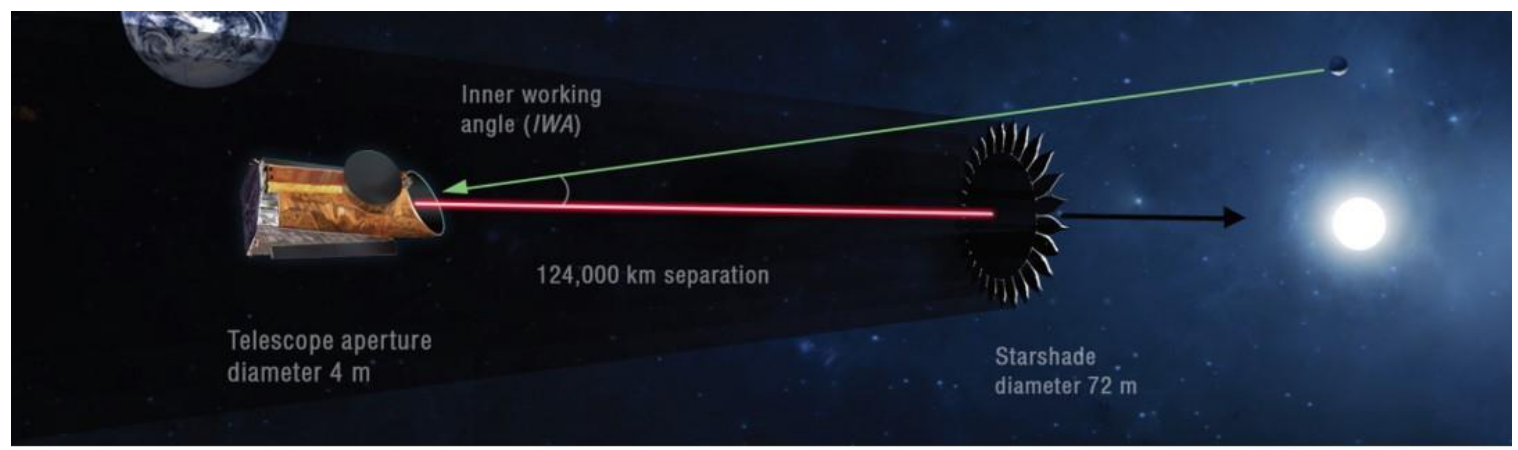

Image from HabEx interim report URS273294

Pre-Decisional - For Planning Purposes Only

Figure 2: Concept for Architecture A

\subsection{HabEx Baseline Telescope Design}

This paper concentrates on the primary mirror for the telescope shown in Figure 3. While the baseline mirror is an openback Zerodur mirror, other considerations such as mass allocations may require alternative designs. This trade study was conducted to get a better understanding of the driving design parameters for a ULE mirror to meet the HabEx criteria.

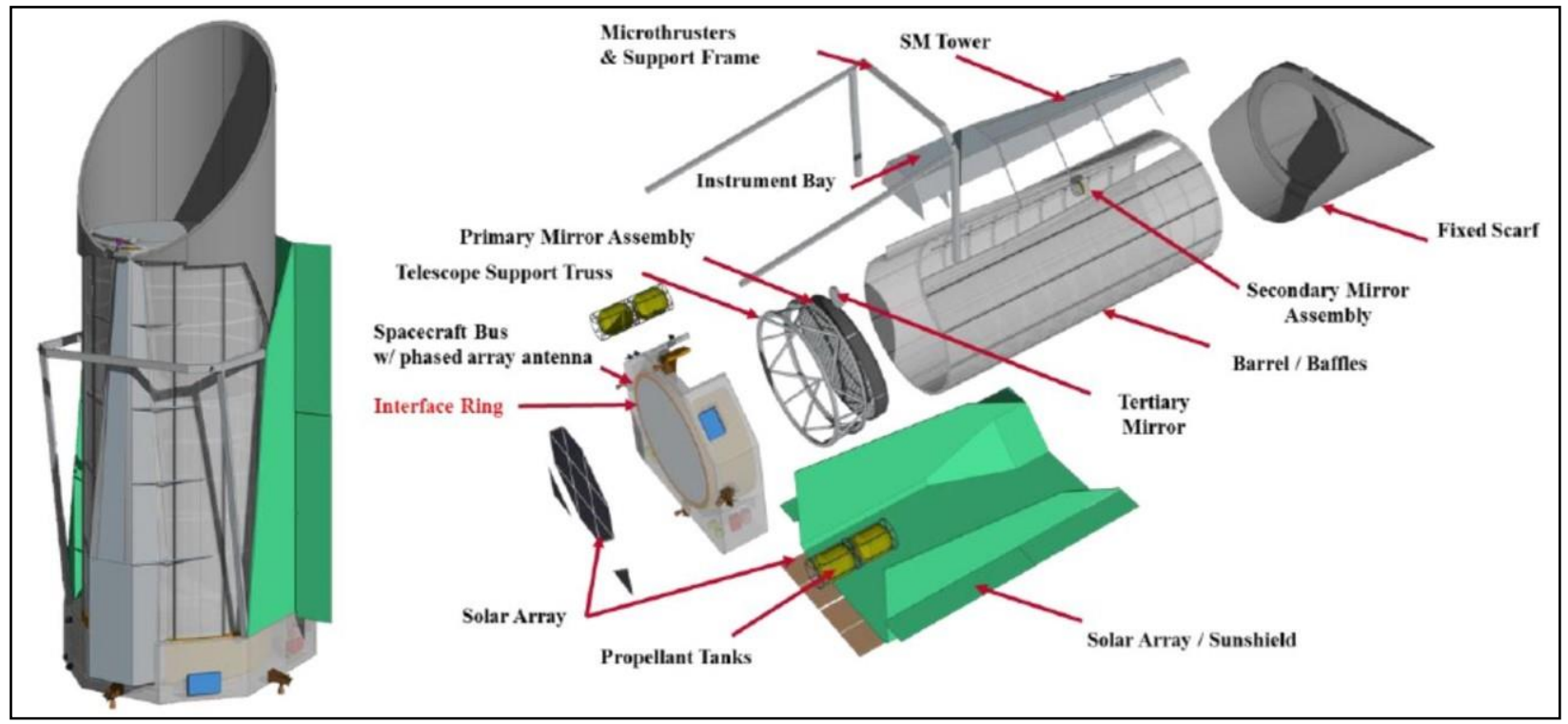

Figure 3: Current Baseline Concept for Telescope portion of satellite

The 4-meter telescope design is off-axis to minimize diffraction, and the station keeping is done using Micro-thrusters, instead of the traditional reaction wheels. The main instrument for planet finding is the advanced coronagraph. Most of the design (mirror performance) requirements are driven by this coronagraph. 


\subsection{HabEx WFE Stability Specification}

The HabEx telescope has a Zernike Polynomial based WFE (wave front error) budget (Figure 4) divided between LOS (line-of-sight) jitter, PM (primary mirror) deformations due to inertial loading, from station keeping, and PM thermal deformations. The LOS jitter is a system level issue (includes secondary mirror and metering structure motions as well a PM motion) and is not addressed in this study. The thermal deformations also depend upon system inputs and are not addressed in this study. The inertial allocation is used to compare trade study results; but remember, the purpose of the study is to understand which parameters influenced WFE, not to find a single point design which meets the specification.

\begin{tabular}{|c|c|c|c|c|c|c|c|c|}
\hline & & & RSS Allocation & $100 \%$ & $1 \%$ & $60 \%$ & $80 \%$ & $10 \%$ \\
\hline \multicolumn{3}{|c|}{ Order } & & VVC-6 Allowable & LOS & Inertial & Thermal & Reserve \\
\hline \multirow[t]{2}{*}{$\mathrm{K}$} & $\mathrm{N}$ & $M$ & Aberration & [pm rms] & [pm rms] & [pm rms] & [pm rms] & [pm rms] \\
\hline & & & TOTAL RMS & 416 & 4 & 250 & 333 & 41 \\
\hline 2 & 1 & 1 & Tilt & & 0 & 0 & 0 & 0 \\
\hline 3 & 2 & 0 & Power (Defocus) & 250 & 2.5 & 150 & 200 & 24.75 \\
\hline 4 & 2 & 2 & Pri Astigmatism & 200 & 2 & 120 & 160 & 19.8 \\
\hline 5 & 3 & 1 & Pri Coma & 175 & 1.75 & 105 & 140 & 17.325 \\
\hline 6 & 4 & 0 & Pri Spherical & 200 & 2 & 120 & 160 & 19.8 \\
\hline 7 & 3 & 3 & Pri Trefoil & 2.6 & 0.026 & 1.56 & 2.08 & 0.2574 \\
\hline 8 & 4 & 2 & Sec Astigmatism & 0.35 & 0.0035 & 0.21 & 0.28 & 0.03465 \\
\hline 9 & 5 & 1 & Sec Coma & 0.35 & 0.0035 & 0.21 & 0.28 & 0.03465 \\
\hline 10 & 6 & 0 & Sec Spherical & 0.35 & 0.0035 & 0.21 & 0.28 & 0.03465 \\
\hline 11 & 4 & 4 & Pri Tetrafoil & 0.35 & 0.0035 & 0.21 & 0.28 & 0.03465 \\
\hline 12 & 5 & 3 & Sec Trefoil & 0.35 & 0.0035 & 0.21 & 0.28 & 0.03465 \\
\hline 13 & 6 & 2 & Ter Astigmatism & 0.1 & 0.001 & 0.06 & 0.08 & 0.0099 \\
\hline 14 & 7 & 1 & Ter Coma & 0.1 & 0.001 & 0.06 & 0.08 & 0.0099 \\
\hline 15 & 8 & 0 & Ter Spherical & 0.1 & 0.001 & 0.06 & 0.08 & 0.0099 \\
\hline 16 & 5 & 5 & Pri Pentafoil & 0.35 & 0.0035 & 0.21 & 0.28 & 0.03465 \\
\hline 17 & 6 & 4 & Sec Tetrafoil & 0.1 & 0.001 & 0.06 & 0.08 & 0.0099 \\
\hline 18 & 7 & 3 & Ter Trefoil & 0.1 & 0.001 & 0.06 & 0.08 & 0.0099 \\
\hline 19 & 8 & 2 & Qua Astigmatism & 0.1 & 0.001 & 0.06 & 0.08 & 0.0099 \\
\hline 20 & 9 & 1 & Qua Coma & 0.1 & 0.001 & 0.06 & 0.08 & 0.0099 \\
\hline 21 & 10 & 0 & Qua Spherical & 0.1 & 0.001 & 0.06 & 0.08 & 0.0099 \\
\hline 22 & 12 & 0 & Qin Spherical & 0.1 & 0.001 & 0.06 & 0.08 & 0.0099 \\
\hline
\end{tabular}

Figure 4: JPL Initial Tolerance Allocation for Primary Mirror

\section{MIRROR TRADE STUDY}

The purpose of the trade study was to determine the mirror and suspension system design parameters which influence the mirror's on-orbit performance. The study varied mirror design parameters of core thickness, core cell size, cell wall thickness and facesheet thickness (Figure 5). For most of the study, the reinforcement thicknesses, pad diameter and pad perimeter diameters were held constant. A sub-study compared the influence of no reinforcement (web thickening) and smaller pad size. The sub-study used the same mesh as the reinforced designs. The study also traded support system variations. The hexapod (kinematic) support has three primary variable: number of attachment pads, location of attachment pads and hexapod strut angles relative to the ground plane. This study compared the performance of three attachment pads on mirror with two legs each versus a six pad configuration with one leg on each pad. The pads were attached to the mirror at $100 \%, 85 \%$ and $70 \%$ of the mirror's radius (Figure 6). Hexapod stiffness determines the ratio of horizontal modes (X and Y) WFE relative to optical direction (Z) WFE. Two angles are significant in hexapod stiffness. The horizontal angle controls the rotation about $\mathrm{Z}$ and together with attachment diameter controls ratio of tilts $\mathrm{X}$ and $\mathrm{Y}$ to loading in a given direction. As one of the input HabEx design goals is a balanced response in all three directions, the horizontal angle was fixed (maximized the rotation about $\mathrm{Z}$ torsional modes) and the hexapod elevation angle (or vertical angle) was varied. The method of varying this parameter in the $\mathrm{AMM}^{4,5}$ was to increase the hexapod height variable while keeping the upper and lower diameters and plan-view angles the same. (To understand how the modeler works, see the references). 


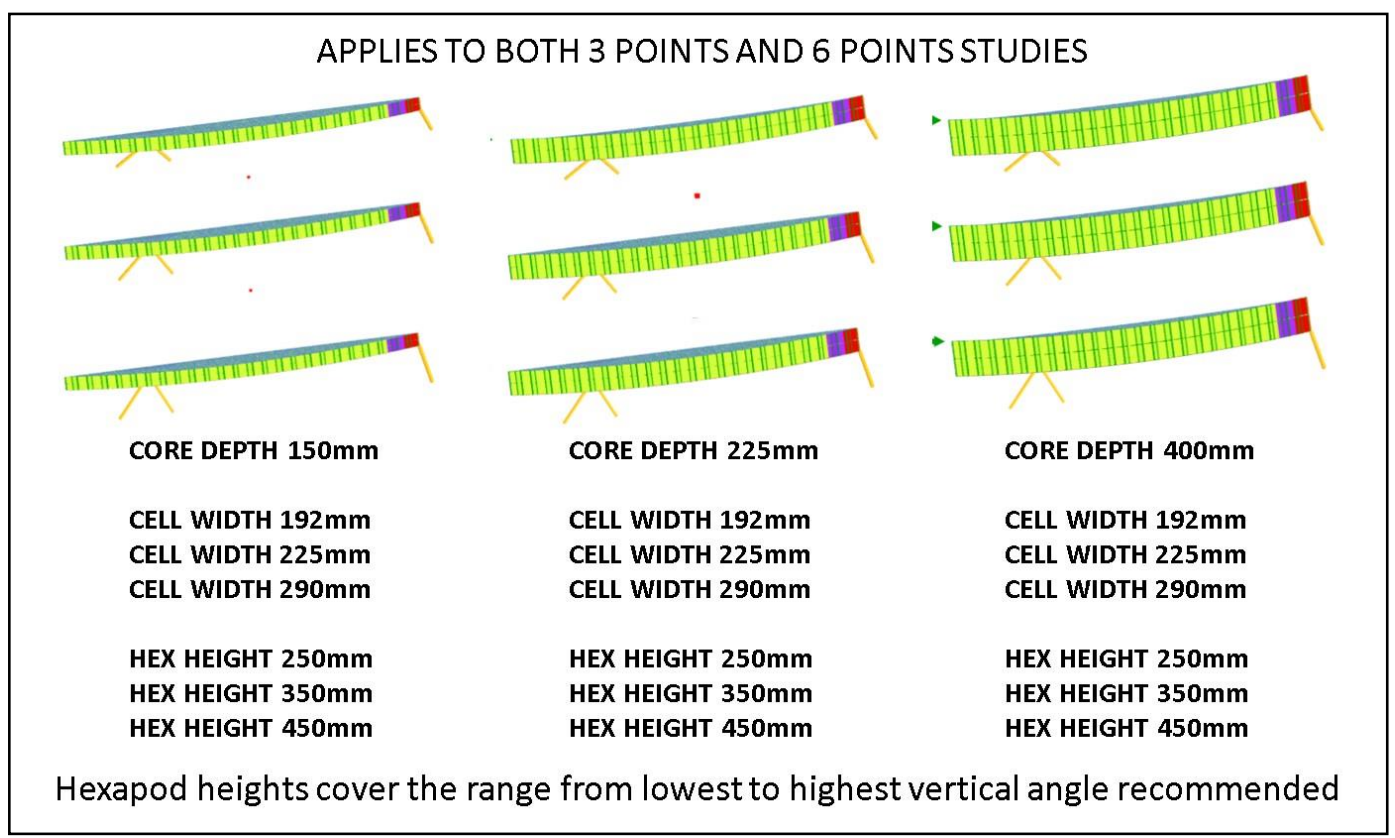

Figure 5: Core Depth and Hexapod Angles

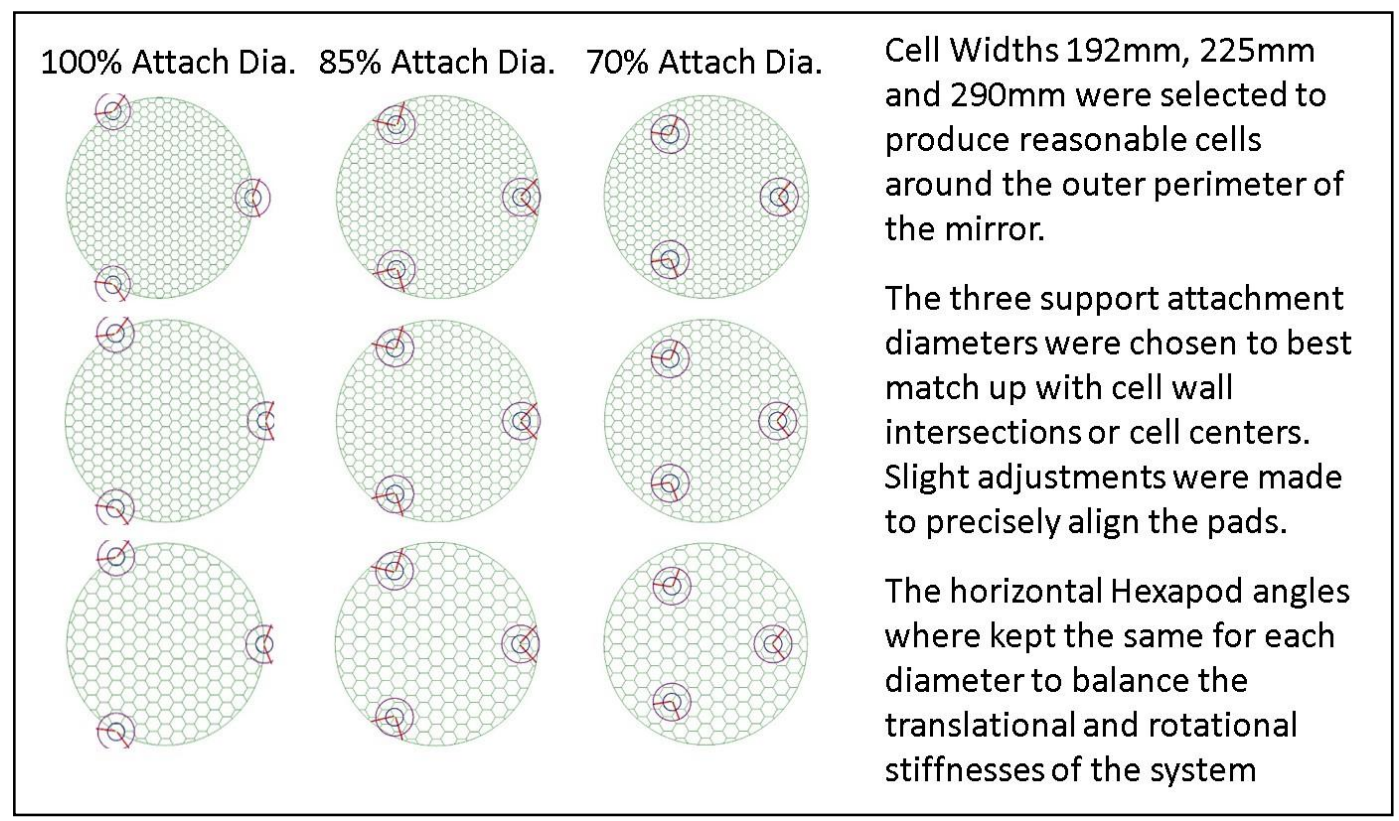

Figure 6: 3 Point Hexapod Geometries

\subsection{Scope of Trade Study}

The AMM (Arnold Mirror Modeler) software was used to generate separate FEM models of each design point. A separate archive file was generated for each point to permit rerunning or expanding the search about a specific design point. The AMM generates an ANSYS APDL which inputs the model, runs $1 \mathrm{~g}$ static acceleration loadcases in X,Y and $\mathrm{Z}$ directions, post processes these results for mean displacements and RMS surface error for each case, then calculates and plots the first 10 supported modes and the first 10 free-free (mirror only) modes. Each run generates a summary file, and displacement files (of the optical surface nodes only) for input into a Zernike decomposition program. Over 264 cases were created, run and post-processed (in this instance, run thru the Zernike Decomp program) in a two weeks span. The longest time was transferring the results in EXCEL for graphs and tables. 


\subsection{Three Points Hexapod Results}

The results are presented in two formats, graphical (Figure 7) and tabular (Figure 8). The thicker core depths produce higher mounted frequencies but at higher mass. Larger core cells reduce the mass with negligible frequency change. The higher mount diameters increase frequencies. Mount vertical angle has negligible effect on first mode frequencies.

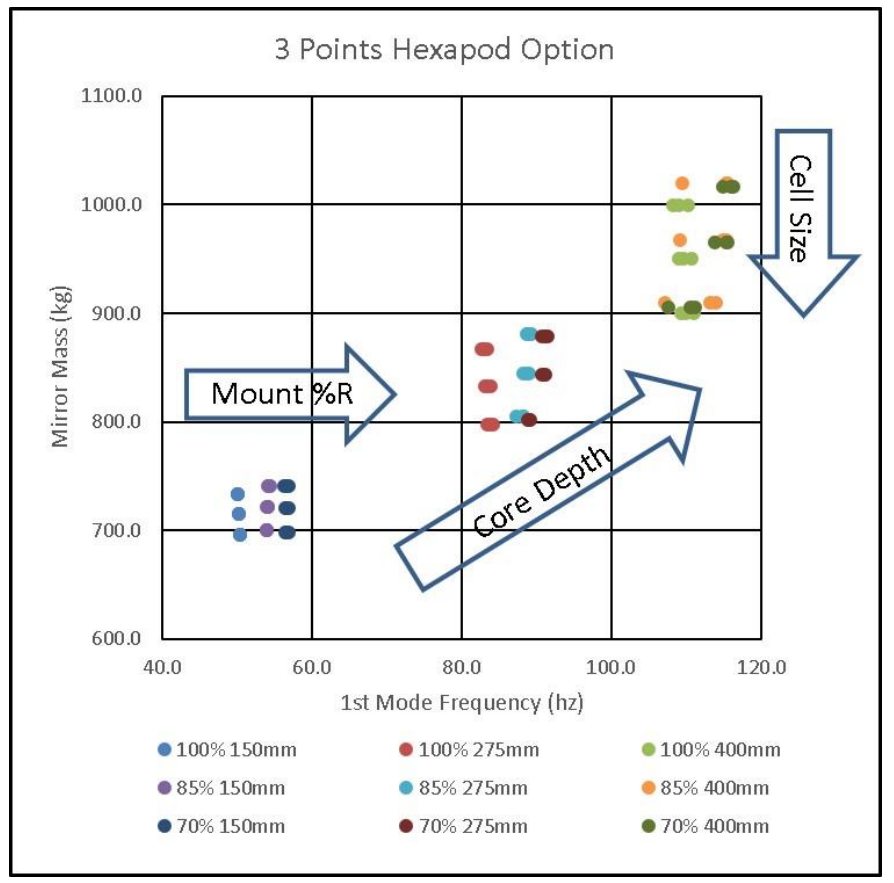

Figure 7: 3 Points Hexapod Mass versus Frequency

\section{0\% Diameter}

\begin{tabular}{|c|c|c|c|c|c|}
\hline HEX & CORE & CELL & SUPPORTS & UPPORT & $X Y Z$ \\
\hline HEIGHT & DEPTH & WIDTH & MASS & 1ST & RMS \\
\hline 0.250 & 0.150 & 0.192 & 733.7 & 50.2 & $3.626 \mathrm{E}-03$ \\
\hline 0.350 & 0.150 & 0.192 & 733.7 & 50.1 & $3.625 \mathrm{E}-03$ \\
\hline 0.450 & 0.150 & 0.192 & 733.7 & 50.1 & $3.639 \mathrm{E}-03$ \\
\hline 0.250 & 0.275 & 0.192 & 866.6 & 83.5 & $1.415 \mathrm{E}-03$ \\
\hline 0.350 & 0.275 & 0.192 & 866.6 & 83.0 & $1.414 \mathrm{E}-03$ \\
\hline 0.450 & 0.275 & 0.192 & 866.6 & 82.6 & $1.403 \mathrm{E}-03$ \\
\hline 0.250 & 0.400 & 0.192 & 999.5 & 110.2 & 8.880E-04 \\
\hline 0.350 & 0.400 & 0.192 & 999.5 & 109.0 & 9.015E-04 \\
\hline 0.450 & 0.400 & 0.192 & 999.5 & 108.2 & 9.009E-04 \\
\hline 0.250 & 0.150 & 0.225 & 715.2 & 50.4 & $3.662 \mathrm{E}-03$ \\
\hline 0.350 & 0.150 & 0.225 & 715.2 & 50.3 & $3.660 \mathrm{E}-03$ \\
\hline 0.450 & 0.150 & 0.225 & 715.2 & 50.3 & $3.674 \mathrm{E}-03$ \\
\hline 0.250 & 0.275 & 0.225 & 832.9 & 83.9 & $1.425 \mathrm{E}-03$ \\
\hline 0.350 & 0.275 & 0.225 & 832.9 & 83.4 & $1.423 \mathrm{E}-03$ \\
\hline 0.450 & 0.275 & 0.225 & 832.9 & 83.1 & $1.412 \mathrm{E}-03$ \\
\hline 0.250 & 0.400 & 0.225 & 950.5 & 110.8 & 8.895E-04 \\
\hline 0.350 & 0.400 & 0.225 & 950.5 & 109.6 & 9.009E-04 \\
\hline 0.450 & 0.400 & 0.225 & 950.5 & 109.0 & 8.996E-04 \\
\hline 0.250 & 0.150 & 0.290 & 696.0 & 50.5 & $3.686 \mathrm{E}-03$ \\
\hline 0.350 & 0.150 & 0.290 & 696.0 & 50.5 & $3.682 \mathrm{E}-03$ \\
\hline 0.450 & 0.150 & 0.290 & 696.0 & 50.4 & $3.696 \mathrm{E}-03$ \\
\hline 0.250 & 0.275 & 0.290 & 797.8 & 84.2 & $1.445 \mathrm{E}-03$ \\
\hline 0.350 & 0.275 & 0.290 & 797.8 & 83.7 & $1.441 \mathrm{E}-03$ \\
\hline 0.450 & 0.275 & 0.290 & 797.8 & 83.4 & 1.429E-03 \\
\hline 0.250 & 0.400 & 0.290 & 899.6 & 111.1 & $9.020 \mathrm{E}-04$ \\
\hline 0.350 & 0.400 & 0.290 & 899.6 & 110.0 & 9.101E-04 \\
\hline 0.450 & 0.400 & 0.290 & 899.6 & 109.4 & 9.075E-04 \\
\hline
\end{tabular}

\section{5\% Diameter}

\begin{tabular}{|c|c|c|c|c|c|}
\hline HEX & CORE & CELL & SUPPORT & UPPORT & $X Y Z$ \\
\hline HEIGHT & DEPTH & WIDTH & MASS & $1 \mathrm{ST}$ & RMS \\
\hline 0.250 & 0.150 & 0.192 & 741.2 & 54.4 & $1.895 \mathrm{E}-03$ \\
\hline & & & & & \\
\hline 0.450 & 150 & & & 4.2 & \\
\hline 0.250 & 275 & 192 & 880.4 & 88.7 & $8.171 \mathrm{E}-04$ \\
\hline 0.350 & 0.275 & 0.192 & 880.4 & 89.3 & $8.178 \mathrm{E}-04$ \\
\hline 0.450 & 0.275 & & 0.4 & 88.9 & \\
\hline 0.250 & 400 & & & & \\
\hline 0.350 & & & 1 & & \\
\hline 0.450 & & & & & \\
\hline & & & & & \\
\hline 0.350 & 0.150 & 25 & 72 & 54.2 & \\
\hline 0.450 & 150 & 5 & & 4.0 & \\
\hline 0.250 & 275 & & & 8.3 & \\
\hline 0.350 & 275 & & & 9.0 & \\
\hline 0.450 & 275 & & & 88.7 & \\
\hline 0.250 & 0.400 & & 9 & 109.2 & $=-04$ \\
\hline 0.350 & 0.400 & & & 114.9 & \\
\hline 0.450 & 0.400 & & 966.9 & 115.3 & \\
\hline & & & & & \\
\hline 0.35 & 150 & 0.2 & & 4.1 & \\
\hline 0.450 & 0.150 & 0.25 & 9.9 & 54.0 & $2.027 \mathrm{E}-03$ \\
\hline 0.250 & 0.275 & & & 87.4 & $8.874 \mathrm{E}-04$ \\
\hline & 275 & & & 3.3 & \\
\hline & & & & & \\
\hline & & & & 107.2 & $6.326 \mathrm{E}-04$ \\
\hline & 0.400 & & 909.9 & 113.2 & $6.108 \mathrm{E}-04$ \\
\hline & 0.400 & 0.290 & 909.9 & 113.9 & $6.032 \mathrm{E}-0$ \\
\hline
\end{tabular}

\section{0\% Diameter}

\begin{tabular}{|c|c|c|c|c|c|}
\hline HEX & CORE & CELL & SUPPORTS & UPPPORT & $X Y Z$ \\
\hline HEIGHT & DEPTH & WIDTH & MASS & $1 \mathrm{ST}$ & RMS \\
\hline 0.250 & \begin{tabular}{|l|}
0.150 \\
\end{tabular} & 0.192 & 740.2 & 56.9 & $1.265 \mathrm{E}-03$ \\
\hline 0.350 & 0.150 & 0.192 & 740.2 & 56.6 & 1.202E-03 \\
\hline 0.450 & 0.150 & 0.192 & 740.2 & 56.4 & $1.144 \mathrm{E}-03$ \\
\hline 0.250 & 0.275 & 0.192 & 878.6 & 91.5 & 6.937E-04 \\
\hline 0.350 & 0.275 & 0.192 & 878.6 & 91.2 & 6.814E-04 \\
\hline 0.450 & 0.275 & 0.192 & 878.6 & 90.8 & 6.585E-04 \\
\hline 0.250 & 0.400 & 0.192 & 1017.1 & 114.9 & $5.284 \mathrm{E}-04$ \\
\hline 0.350 & 0.400 & 0.192 & 1017.1 & 116.3 & 5.287E-04 \\
\hline 0.450 & 0.400 & 0.192 & 1017.1 & 116.0 & $5.275 \mathrm{E}-04$ \\
\hline 0.250 & 0.150 & 0.225 & 720.9 & 57.0 & 1.293E-03 \\
\hline 0.350 & 0.150 & 0.225 & 720.9 & 56.7 & $1.234 \mathrm{E}-03$ \\
\hline 0.450 & 0.150 & 0.225 & 720.9 & 56.5 & 1.179E-03 \\
\hline 0.250 & 0.275 & 0.225 & 843.3 & 91.2 & $6.965 \mathrm{E}-04$ \\
\hline 0.350 & 0.275 & 0.225 & 843.3 & 91.0 & 6.849E-04 \\
\hline 0.450 & 0.275 & 0.225 & 843.3 & 90.7 & $6.622 \mathrm{E}-04$ \\
\hline 0.250 & 0.400 & 0.225 & 965.7 & 113.8 & 5.234E-04 \\
\hline 0.350 & 0.400 & 0.225 & 965.7 & 115.5 & $5.257 \mathrm{E}-04$ \\
\hline 0.450 & 0.400 & 0.225 & 965.7 & 115.4 & 5.249E-04 \\
\hline 0.250 & 0.150 & 0.290 & 698.4 & 56.9 & 1.347E-03 \\
\hline 0.350 & 0.150 & 0.290 & 698.4 & 56.7 & $1.278 \mathrm{E}-03$ \\
\hline 0.450 & 0.150 & 0.290 & 698.4 & 56.6 & $1.216 \mathrm{E}-03$ \\
\hline 0.250 & 0.275 & 0.290 & 802.1 & 88.8 & 7.314E-04 \\
\hline 0.350 & 0.275 & 0.290 & 802.1 & 89.2 & 7.173E-04 \\
\hline 0.450 & 0.275 & 0.290 & 802.1 & 89.0 & 6.927E-04 \\
\hline 0.250 & 0.400 & 0.290 & 905.8 & 107.6 & $5.478 \mathrm{E}-04$ \\
\hline 0.350 & 0.400 & 0.290 & 905.8 & 110.6 & 5.523E-04 \\
\hline 0.450 & 0.400 & 0.290 & 905.8 & 111.2 & $5.506 \mathrm{E}-04$ \\
\hline
\end{tabular}

Figure 8: Displacement results for 3 Points Hexapods 


\subsection{Six Points Hexapod Results}

The Six Points Hexapods (Figure 9) had similar trends (Figures 10 and 11), core thickness increase mass and stiffness (as measured by first modal frequency) and cell size decreases mass with small stiffness effects. But, the six-point mount results in greater frequency spread with mount geometry changes. Increases in hexapod height (i.e. vertical mount leg angle) decreases first modal frequency.

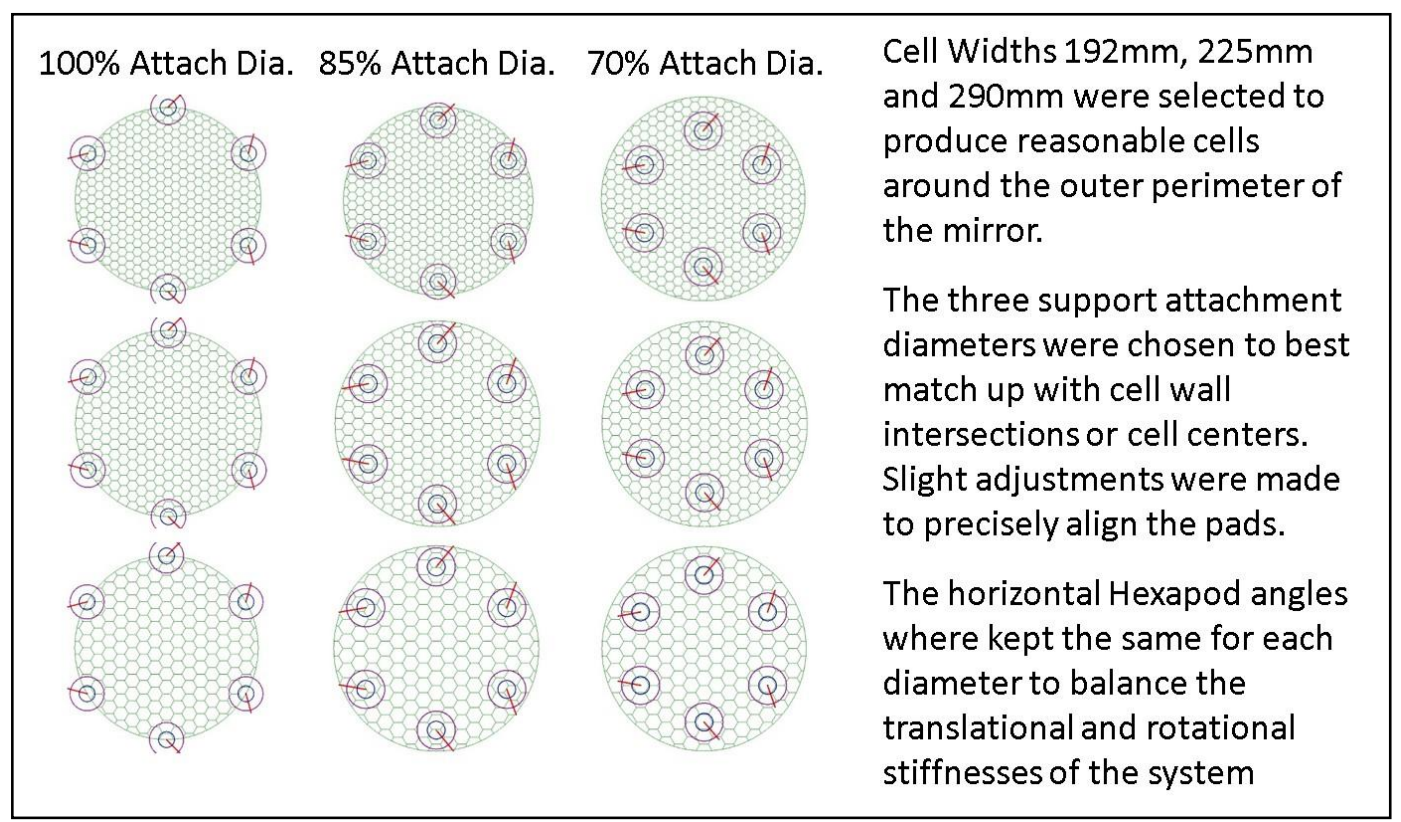

Figure 9: Six Points Hexapod Geometries

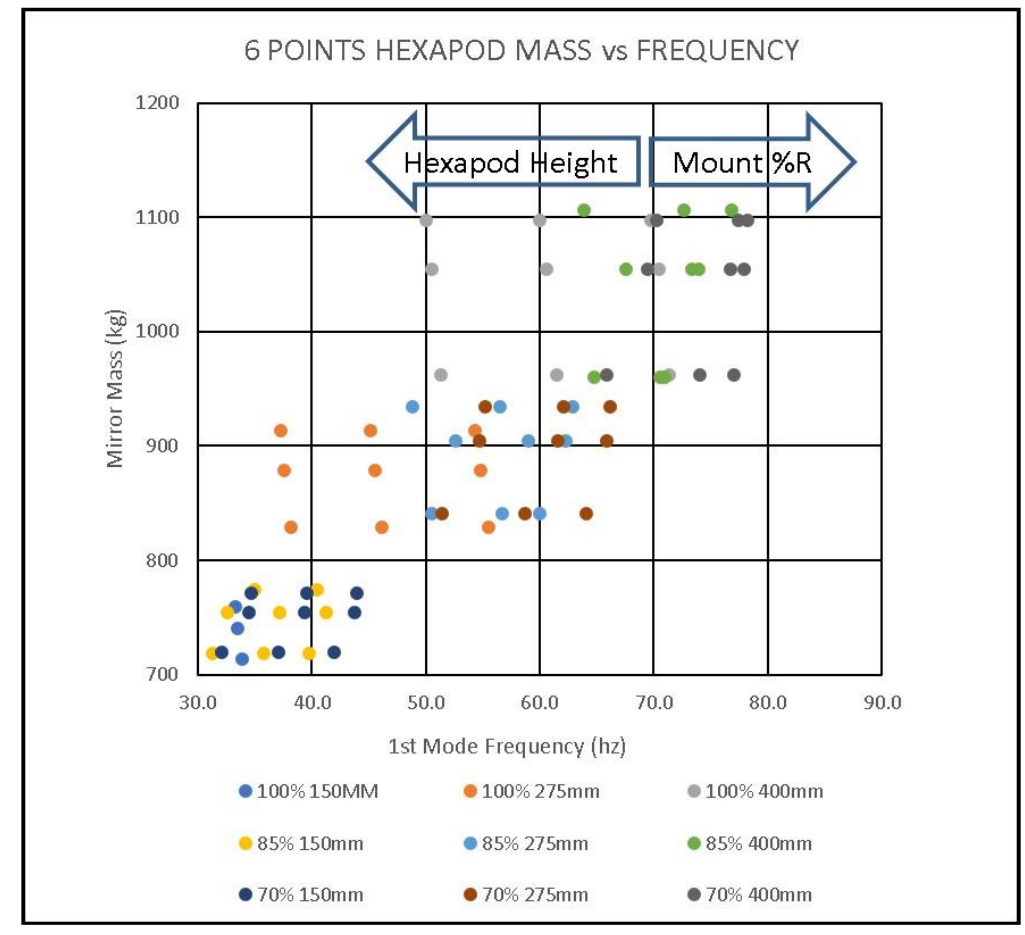

Figure 10: Six Points Hexapod mass versus Frequency 


\begin{tabular}{|c|c|c|c|c|c|c|c|c|c|c|c|c|c|c|c|c|c|}
\hline \multicolumn{6}{|c|}{ 100\% Diameter } & \multicolumn{6}{|c|}{ 85\% Diameter } & \multicolumn{6}{|c|}{ 70\% Diameter } \\
\hline HEX & CORE & CELL & JUPPOR & TUPPOR & $\mathrm{XYZ}$ & HEX & CORE & CELL & SUPPOR & UPPOR & $X Y Z$ & HEX & CORE & CELL & SUPPOR & SUPPOR & $X Y Z$ \\
\hline HEIGHT & DEPTH & WIDTH & MASS & \begin{tabular}{|l|}
$1 S T$ \\
\end{tabular} & RMS & HEIGHT & DEPTH & WIDTH & MASS & 1ST & RMS & HEIGHT & DEPTH & WIDTH & MASS & 1ST & RMS \\
\hline 0.250 & 0.150 & 0.192 & 759.6 & 33.3 & $1.754 \mathrm{E}-02$ & 0.250 & 0.150 & 0.192 & $\begin{array}{l}773.9 \\
\end{array}$ & 40.4 & $1.094 \mathrm{E}-02$ & 0.250 & 0.150 & \begin{tabular}{|l|}
0.192 \\
\end{tabular} & 770.8 & 44.0 & 8.112E-03 \\
\hline 0.350 & 0.150 & 0.192 & 759.6 & 27.0 & $2.509 \mathrm{E}-02$ & 0.350 & 0.150 & 0.192 & 773.9 & 35.0 & $1.584 \mathrm{E}-02$ & 0.350 & 0.150 & 0.192 & 770.8 & 39.6 & 1.188E-02 \\
\hline 0.450 & 0.150 & 0.192 & 759.6 & 22.2 & $3.276 \mathrm{E}-02$ & 0.450 & 0.150 & 0.192 & 773.9 & 29.8 & \begin{tabular}{|l|l} 
& $2.083 E-02$ \\
\end{tabular} & 0.450 & 0.150 & 0.192 & 770.8 & 34.7 & $1.572 \mathrm{E}-02$ \\
\hline 0.250 & 275 & 0.192 & 913.6 & 54.3 & $6.193 \mathrm{E}-03$ & & 0.275 & & 939.9 & & & & 0.275 & & & 66.2 & \\
\hline 0.350 & & 192 & 913.6 & 45.2 & & 0.350 & 0.275 & 192 & 939.9 & 56.5 & $5.755 \mathrm{E}$ & 0.350 & 0.275 & 0.192 & 934.2 & 62.1 & EE-03 \\
\hline 0.450 & 275 & .192 & 913.6 & 37.3 & 1.16 & 0.4 & 0.275 & 192 & 939.9 & 48.9 & & & 0.275 & 0.192 & 934.2 & 55.2 & \\
\hline 0.250 & 400 & .192 & 1067.6 & 69.8 & & & 0.400 & 192 & 1105.9 & 76.8 & & 250 & 0.400 & 0.192 & 1097.6 & 78.2 & \\
\hline 0.350 & 0.400 & 0.192 & 1067.6 & 60.0 & & 50 & 0.400 & 0.192 & 11 & 72.6 & & 0.350 & 0.400 & 0.192 & 1097.6 & 77.5 & \\
\hline 0.450 & 0.400 & 0.192 & 067.6 & 50.1 & 6.4 & 0.450 & 0.400 & 0.192 & 1105.9 & 63.9 & 4.315 & 0.450 & 0.400 & 0.192 & 1097.6 & 70.3 & 3.5 \\
\hline & & & & & & & & & & & & & & & & & \\
\hline 0.250 & 0.150 & 0.225 & 740.2 & 33.5 & $1.749 \mathrm{E}-02$ & 0.250 & 0.150 & 0.225 & 754.7 & 41.3 & 9.5 & 0.250 & 0.150 & 0.225 & 754.7 & 43.8 & 8.231E-03 \\
\hline 0.350 & 0.150 & 0.225 & 740.2 & 27.2 & $2.501 \mathrm{E}-02$ & 0.350 & 0.150 & 0.225 & 754.7 & 37.2 & & 0.350 & 0.150 & 0.225 & 754.7 & 39.4 & 1.20 \\
\hline 0.450 & 0.150 & 0.225 & 740.2 & 22.3 & $3.265 \mathrm{E}-02$ & 0.450 & 0.150 & 0.225 & 754.7 & 32.6 & & 0.450 & 0.150 & 0.225 & 754.7 & 34.5 & $\mathrm{E}-02$ \\
\hline 0.250 & 0.275 & 0.225 & 878.1 & 54.8 & $6.150 \mathrm{E}-03$ & 0.250 & 0.275 & 0.225 & 904.7 & 62.3 & $3.536 \mathrm{E}-03$ & 0.250 & 0.275 & 0.225 & 904.7 & 65.9 & $3.129 \mathrm{E}-03$ \\
\hline 0.350 & 0.275 & 0.225 & 878.1 & 45.6 & $8.767 \mathrm{E}-03$ & 0.350 & 0.275 & 0.225 & 904.7 & 59.0 & 5.127E-03 & & 0.275 & 0.225 & 904.7 & 61.6 & \\
\hline 0.450 & 275 & .225 & 878.1 & 37.6 & & 0.450 & .275 & 225 & 904.7 & 52.6 & & & & & & 54.7 & \\
\hline 0.250 & 400 & 0.225 & 016.0 & 70.4 & & & 400 & 0.225 & 1054.8 & 73.3 & & & 0.400 & 0.225 & & 77.9 & \\
\hline 0.350 & 0.400 & 0.225 & 1016.0 & 60.6 & & & 0.400 & 0.225 & 10 & 73.9 & 2.9 & 0.350 & 0.400 & 0.225 & 1054.8 & 76.8 & $2.716 \mathrm{E}-03$ \\
\hline 0.450 & 0.400 & 0.225 & 1016.0 & 50.5 & $6.341 \mathrm{E}-03$ & 0.450 & 0.400 & 0.225 & 1054.8 & 67.6 & $3.936 \mathrm{E}-0$ & 0.450 & 0.400 & 0.225 & 1054.8 & 69.5 & $3.626 \mathrm{E}-03$ \\
\hline & & & & & & & & & & & & & & & & & \\
\hline 0.250 & 0.150 & 0.290 & 713.4 & 33.9 & $1.756 \mathrm{E}-02$ & 0.250 & 0.150 & 0.290 & 718.9 & 39.8 & $1.054 \mathrm{E}-02$ & 0.250 & 0.150 & 0.290 & 719.8 & 42.0 & $9.615 \mathrm{E}-03$ \\
\hline 0.350 & 0.150 & 0.290 & 713.4 & 27.5 & $2.507 \mathrm{E}-02$ & 0.350 & 0.150 & 0.290 & 718.9 & 35.8 & $1.536 \mathrm{E}-02$ & 0.350 & 0.150 & 0.290 & 719.8 & 37.1 & $1.403 \mathrm{E}-02$ \\
\hline 0.450 & 0.150 & 0.290 & 713.4 & 22.5 & $3.272 \mathrm{E}-02$ & 0.450 & 0.150 & 0.290 & 718.9 & 31.3 & $2.028 \mathrm{E}-02$ & 0.450 & 0.150 & 0.290 & 719.8 & 32.1 & 1.851E-02 \\
\hline 0.250 & 0.275 & 0.290 & 829.2 & 55.5 & $6.129 \mathrm{E}-03$ & 0.250 & 0.275 & 0.290 & 839.3 & 60.0 & $3.921 \mathrm{E}-03$ & 0.250 & 0.275 & 0.290 & 840.8 & 64.1 & $3.616 \mathrm{E}-03$ \\
\hline 0.350 & 0.275 & 0.290 & 829.2 & 46.2 & $8.723 E-03$ & 0.350 & 0.275 & 0.290 & 839.3 & 56.7 & 5.651E-03 & 0.350 & 0.275 & 0.290 & 840.8 & 58.7 & $5.280 \mathrm{E}-03$ \\
\hline 0.450 & 0.275 & 0.290 & 829.2 & 38.2 & $1.142 \mathrm{E}-02$ & 0.450 & 0.275 & 0.290 & 839.3 & 50.5 & $7.466 \mathrm{E}-03$ & 0.450 & 0.275 & 0.290 & 840.8 & 51.4 & $6.998 \mathrm{E}-03$ \\
\hline 0.250 & 0.400 & 0.290 & 945.0 & 71.4 & $3.423 \mathrm{E}-03$ & 0.250 & 0.400 & 0.290 & 959.6 & 70.6 & $2.278 \mathrm{E}-03$ & 0.250 & 0.400 & 0.290 & 961.9 & 77.1 & $2.108 \mathrm{E}-03$ \\
\hline 0.350 & 0.400 & 0.290 & 945.0 & 61.5 & $4.802 \mathrm{E}-03$ & 0.350 & 0.400 & 0.290 & 959.6 & 71.0 & & 0.350 & 0.400 & 0.290 & 961.9 & 74.0 & 3.100E-03 \\
\hline 0.450 & 0.400 & 0.290 & 945.0 & 51.3 & $6.272 \mathrm{E}-03$ & 0.450 & 0.400 & 0.290 & 959.6 & 64.8 & $4.334 \mathrm{E}-03$ & 0.450 & 0.400 & 0.290 & 961.9 & 65.9 & 4.115E-03 \\
\hline
\end{tabular}

Figure 11: Displacement results for Six Points Hexapods

\section{INERTIAL DEFORMATION}

To our knowledge, inertial deformation may be a new performance metric and is defined as the mirror deformation produced by an acceleration. For example, gravity sag is the deformation produced when the mirror (on its mount) is exposed to a 1-G acceleration. On-orbit, inertial deformation is the response of the mirror (reacting against its mount) when it is exposed to accelerations from sources such slews, solar wind, reaction wheel noise, etc. And, can be modeled simply as gravity sag deformation scaled by the ratio of accelerations. In the case of HabEx, point stability will be maintained via micro-thrusters with a specified noise of 0.1 micro-Newton $(\sim 0.01$ micro-G). This error is then decomposed into Zernike polynomials and compared to the specified error budget.

\subsection{Analysis Process}

Due to numerical accuracy inherent in finite element modeling and types of models and elements used, all analyses were performed in meters at one gravity $\left(9.802 \mathrm{~m} / \mathrm{s}^{\wedge} 2\right)$, the displacements were then scaled to the appropriate levels. The load-cases were unit $1 \mathrm{~g}$ accelerations in the three axes (X, Y and Z) (Figure 12). These displacements were output into individual files and then input into a purpose-built Zernike Decomposition program which processed each load-case. Zernikes were scaled to the appropriate units then RSSed (square root of the sum of squares) together (Figure 13) to be compared to the tolerance table. The dedicated program created a results file for insertion into EXCEL tables reported in Figures 14, 15 and 21. 


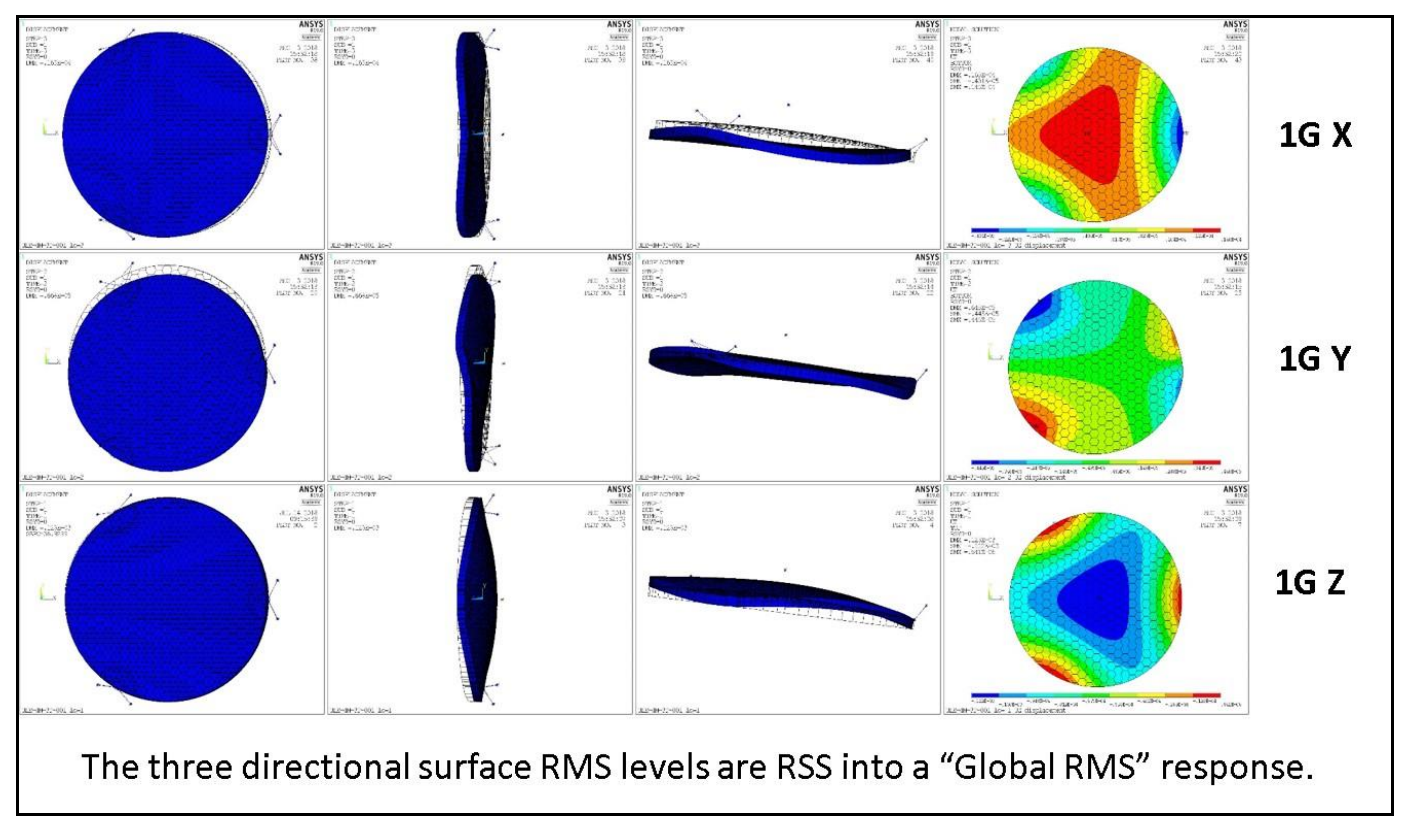

Figure 12: Typical Static Load-Case Results

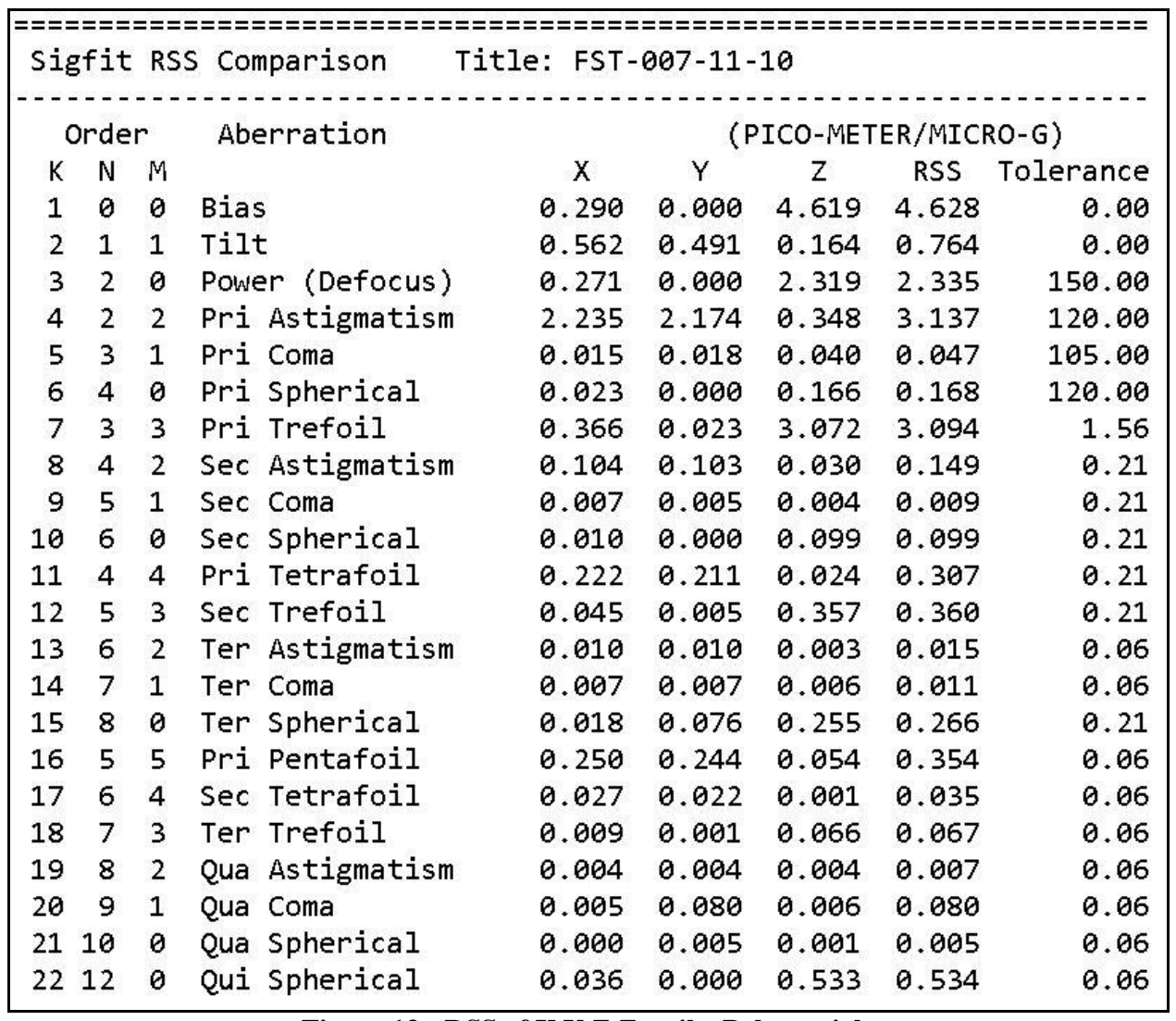

Figure 13: RSS of X,Y,Z Zernike Polynomials 


\subsection{Core Depth Trade Study}

This portion of the study looked at one cell size and the $100 \%$ mount diameter. The main result was that none of the variations met the WFE error budget specification in all terms. But, the thicker and stiffer the mirror, the better.

\begin{tabular}{|c|c|c|c|c|c|c|c|}
\hline \multirow{2}{*}{$\frac{\text { ZERNIKES }}{\mathrm{KN} \mathrm{M}}$} & \multirow{2}{*}{$\begin{array}{l}\text { WFE Tol } \\
\text { [pm rms] }\end{array}$} & \multicolumn{3}{|c|}{3 Point Mount Error [pm rms] } & \multicolumn{3}{|c|}{6 Point Mount Error [pm rms] } \\
\hline & & $150 \mathrm{~mm}$ & $275 \mathrm{~mm}$ & $400 \mathrm{~mm}$ & $150 \mathrm{~mm}$ & $275 \mathrm{~mm}$ & $400 \mathrm{~mm}$ \\
\hline 320 Power (Defocus) & 150.00 & 11.376 & 4.238 & 2.335 & 11.067 & 3.822 & 1.939 \\
\hline 422 Pri Astigmatism & 120.00 & 1.128 & 3.037 & 3.033 & 60.995 & 19.948 & 9.967 \\
\hline 531 Pri Coma & 105.00 & 0.252 & 0.083 & 0.047 & 0.253 & 0.104 & 0.071 \\
\hline $6 \quad 4 \quad 0$ Pri Spherical & 120.00 & 0.520 & 0.258 & 0.166 & 0.797 & 0.373 & 0.233 \\
\hline 733 Pri Trefoil & 1.56 & 14.761 & 5.431 & 3.094 & 1.597 & 1.225 & 1.025 \\
\hline $842 \mathrm{Sec}$ Astigmatism & 0.21 & 0.051 & 0.120 & 0.144 & 2.718 & 1.094 & 0.669 \\
\hline $951 \mathrm{Sec}$ Coma & 0.21 & 0.022 & 0.004 & 0.009 & 0.017 & 0.007 & 0.016 \\
\hline $10 \quad 6 \quad 0$ Sec Spherical & 0.21 & 0.334 & 0.146 & 0.097 & 0.252 & 0.132 & 0.102 \\
\hline 1144 Pri Tetrafoil & 0.21 & 0.491 & 0.384 & 0.302 & 5.996 & 2.336 & 1.404 \\
\hline $1253 \mathrm{Sec}$ Trefoil & 0.21 & 1.171 & 0.527 & 0.352 & 0.120 & 0.108 & 0.100 \\
\hline 1362 Ter Astigmatism & 0.06 & 0.029 & 0.016 & 0.014 & 0.224 & 0.151 & 0.124 \\
\hline 1471 Ter Coma & 0.06 & 0.026 & 0.011 & 0.011 & 0.019 & 0.010 & 0.012 \\
\hline 1580 Ter Spherical & 0.06 & 1.213 & 0.454 & 0.266 & 0.671 & 0.295 & 0.208 \\
\hline 1655 Pri Pentafoil & 0.21 & 0.365 & 0.377 & 0.342 & 0.693 & 0.528 & 0.443 \\
\hline 1764 Sec Tetrafoil & 0.06 & 0.055 & 0.047 & 0.035 & 0.749 & 0.418 & 0.318 \\
\hline 1873 Ter Trefoil & 0.06 & 0.044 & 0.065 & 0.064 & 0.016 & 0.008 & 0.003 \\
\hline 1982 Qua Astigmatism & 0.06 & 0.012 & 0.005 & 0.007 & 0.033 & 0.022 & 0.017 \\
\hline 2091 Qua Coma & 0.06 & 0.097 & 0.088 & 0.078 & 0.182 & 0.084 & 0.053 \\
\hline 21102 Qua Astigmatism & 0.06 & 0.003 & 0.003 & 0.005 & 0.003 & 0.002 & 0.003 \\
\hline 22120 Qin Spherical & 0.06 & 2.613 & 0.943 & 0.536 & 1.350 & 0.540 & 0.373 \\
\hline
\end{tabular}

Figure 14: Processed results of Core Depth Study

\subsection{Mount Diameter Trade Study}

This portion looked at the 400mm Core Depth and 290mm Cell Size and varied the mount diameters for 3 and 6 point cases. Different mount pad and radial distance locations result is different Zernike polynomial WFE distributions.

\begin{tabular}{|c|c|c|c|c|c|c|c|}
\hline \multirow{2}{*}{$\frac{\text { ZERNIKES }}{\mathrm{K} \mathrm{N} \mathrm{M}}$} & \multirow{2}{*}{$\begin{array}{r}\text { WFE Tol } \\
\text { [pm rms] }\end{array}$} & \multicolumn{3}{|c|}{3 Point Mount Error [pm rms] } & \multicolumn{3}{|c|}{6 Point Mount Error [pm rms] } \\
\hline & & $100 \%$ & $85 \%$ & $70 \%$ & $100 \%$ & $85 \%$ & $70 \%$ \\
\hline 320 Power (Defocus) & 150.00 & 2.599 & 0.632 & 0.834 & 2.226 & 0.083 & 0.633 \\
\hline 422 Pri Astigmatism & 120.00 & 2.876 & 2.813 & 2.633 & 9.527 & 4.727 & 5.050 \\
\hline 531 Pri Coma & 105.00 & 0.050 & 0.231 & 0.491 & 0.069 & 0.357 & 0.478 \\
\hline 640 Pri Spherical & 120.00 & 0.031 & 0.365 & 0.244 & 0.136 & 0.451 & 0.325 \\
\hline 733 Pri Trefoil & 1.56 & 3.129 & 2.397 & 1.608 & 0.969 & 0.788 & 0.589 \\
\hline $842 \mathrm{Sec}$ Astigmatism & 0.21 & 0.135 & 0.361 & 0.639 & 0.684 & 1.371 & 1.807 \\
\hline $951 \mathrm{Sec}$ Coma & 0.21 & 0.013 & 0.048 & 0.047 & 0.021 & 0.039 & 0.023 \\
\hline $1060 \mathrm{Sec}$ Spherical & 0.21 & 0.060 & 0.006 & 0.258 & 0.067 & 0.023 & 0.222 \\
\hline 1144 Pri Tetrafoil & 0.21 & 0.289 & 0.085 & 0.022 & 1.457 & 0.953 & 0.895 \\
\hline $1253 \mathrm{Sec}$ Trefoil & 0.21 & 0.397 & 1.169 & 1.243 & 0.093 & 0.182 & 0.188 \\
\hline $13 \quad 6 \quad 2$ Ter Astigmatism & 0.06 & 0.019 & 0.012 & 0.166 & 0.150 & 0.064 & 0.361 \\
\hline 1471 Ter Coma & 0.06 & 0.013 & 0.023 & 0.064 & 0.017 & 0.041 & 0.066 \\
\hline 1580 Ter Spherical & 0.06 & 0.382 & 0.208 & 0.174 & 0.278 & 0.160 & 0.083 \\
\hline 1655 Pri Pentafoil & 0.21 & 0.345 & 0.247 & 0.151 & 0.437 & 0.319 & 0.284 \\
\hline $1764 \mathrm{Sec}$ Tetrafoil & 0.06 & 0.029 & 0.050 & 0.046 & 0.354 & 0.810 & 0.971 \\
\hline 1873 Ter Trefoil & 0.06 & 0.083 & 0.122 & 0.509 & 0.002 & 0.030 & 0.051 \\
\hline 1982 Qua Astigmatism & 0.06 & 0.009 & 0.038 & 0.020 & 0.015 & 0.169 & 0.127 \\
\hline 2091 Qua Coma & 0.06 & 0.077 & 0.039 & 0.027 & 0.068 & 0.013 & 0.018 \\
\hline 21102 Qua Astigmatism & 0.06 & 0.009 & 0.005 & 0.022 & 0.005 & 0.002 & 0.014 \\
\hline 22120 Qin Spherical & 0.06 & 0.857 & 0.629 & 0.491 & 0.639 & 0.484 & 0.414 \\
\hline
\end{tabular}

Figure15: Mount Diameter Trade Study 


\subsection{Trade Study on Back Profiles}

This study investigated the influence of the back profile. Based upon earlier open-backed results we were expecting an effect. But, for the closed back design, the back-sheet seems to have negated the benefit of a deeper outer zone.

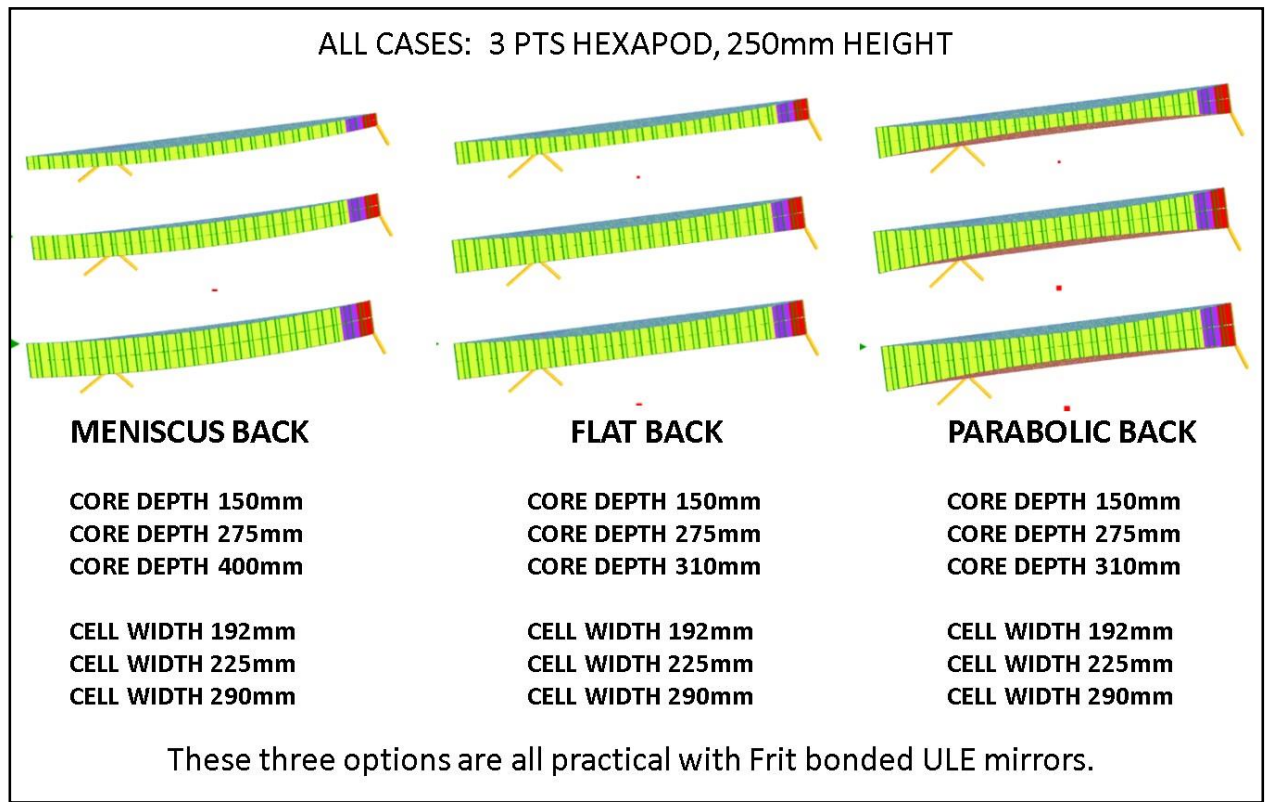

Figure 16: Basic Parameters looked at in Back Profile Study.

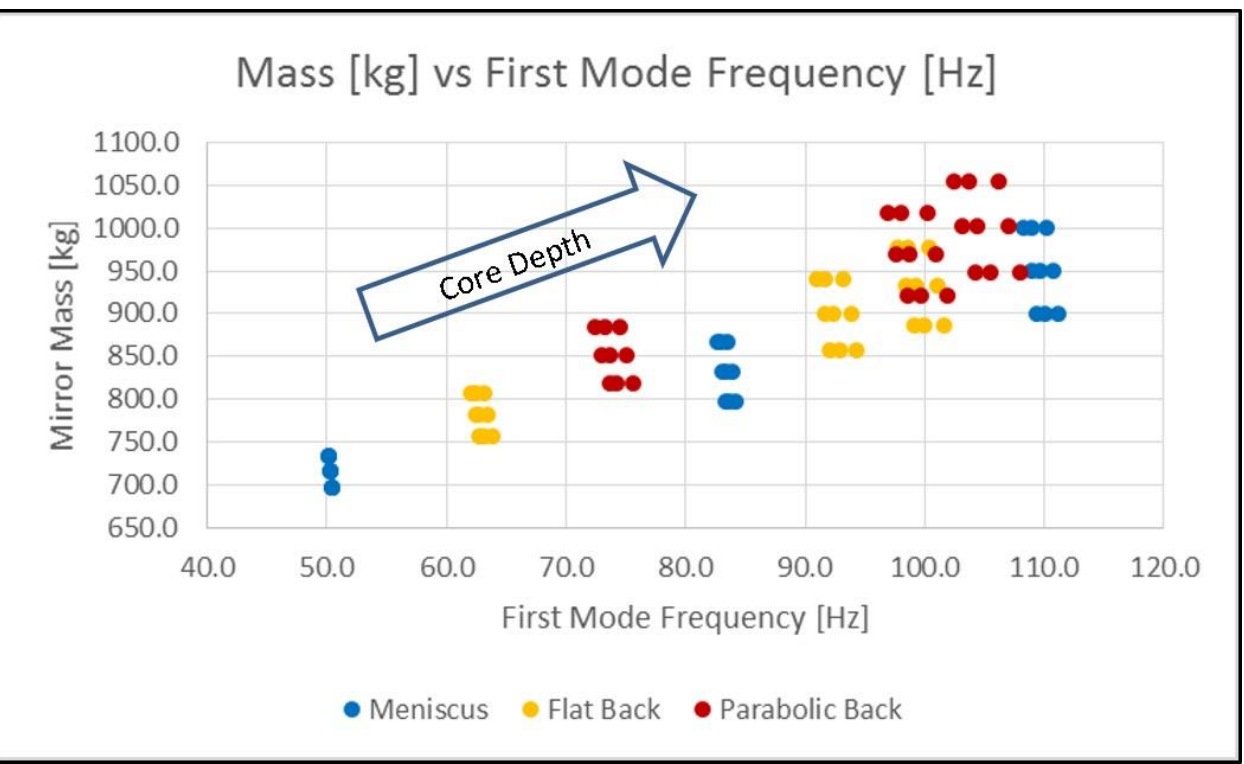

Figure 17: Mass versus Frequency for Back Profiles. 


\begin{tabular}{|c|c|c|c|c|c|c|c|c|c|c|c|c|c|c|c|c|c|}
\hline \multicolumn{6}{|c|}{ MENISCUS } & \multicolumn{6}{|c|}{ FLAT BACK } & \multicolumn{6}{|c|}{ PARABOLIC BACK } \\
\hline HEX & CORE & CELL & SUPPORTS & UPPORT & $X Y Z$ & HEX & CORE & CELL & SUPPORTS & UPPORT & $\mathrm{XYZ}$ & HEX & CORE & CELL & SUPPORTS & SUPPORT & $X Y Z$ \\
\hline HEIGHT & DEPTH & WIDTH & MASS & $1 \mathrm{ST}$ & RMS & HEIGHT & DEPTH & WIDTH & MASS & 1ST & RMS & HEIGHT & DEPTH & WIDTH & MASS & $1 \mathrm{ST}$ & RMS \\
\hline 0.250 & 0.150 & \begin{tabular}{|l|}
0.192 \\
\end{tabular} & 733.7 & 50.2 & $3.626 \mathrm{E}-03$ & 0.250 & 0.150 & 0.192 & \begin{tabular}{|r|}
807.8 \\
\end{tabular} & 63.2 & $4.168 \mathrm{E}-03$ & 0.250 & 0.150 & 0.192 & 883.9 & 74.5 & 2.647E-03 \\
\hline 0.350 & 0.150 & 0.192 & 733.7 & 50.1 & $3.625 \mathrm{E}-03$ & 0.350 & 0.150 & 0.192 & 807.8 & 62.5 & $4.300 \mathrm{E}-03$ & 0.350 & 0.150 & 0.192 & 883.9 & 73.2 & $2.805 \mathrm{E}-03$ \\
\hline 0.450 & 0.150 & 0.192 & 733.7 & 50.1 & 3.639E-03 & 0.450 & 0.150 & 0.192 & 807.8 & 62.1 & $4.382 \mathrm{E}-03$ & 0.450 & & & 883.9 & 72.5 & $2.906 \mathrm{E}-03$ \\
\hline 0.250 & & 0.192 & 866.6 & 83.5 & & 0.250 & 0.275 & 0.192 & 940.7 & \begin{tabular}{l|l|}
93.2 \\
\end{tabular} & $1.911 \mathrm{E}-03$ & 0.250 & 0.275 & & \begin{tabular}{|l|}
1016.9 \\
\end{tabular} & 100.3 & \\
\hline 0.350 & 0.275 & 0.192 & 866.6 & 83.0 & $1.414 \mathrm{E}-03$ & 0.350 & 0.275 & 0.192 & 940.7 & 91.7 & 1.978E-03 & & & & & 98.0 & \\
\hline 0.450 & 0.275 & 0.192 & 866.6 & 82.6 & $1.403 \mathrm{E}-03$ & 0.450 & 0.275 & 0.192 & 940.7 & 90.9 & $2.026 \mathrm{E}-03$ & 0.450 & 0.275 & 0.192 & 1016.9 & 96.9 & $1.578 \mathrm{E}-03$ \\
\hline 0.250 & 0.400 & 0.192 & 999.5 & 110.2 & \begin{tabular}{|l|}
$8.880 \mathrm{E}-04$ \\
\end{tabular} & 0.250 & 0.310 & 0.192 & 977.9 & 100.4 & 1.647E-03 & 0.250 & 0.310 & 0.192 & 1054.1 & 106.2 & $1.278 \mathrm{E}-03$ \\
\hline 0.350 & 0.400 & 0.192 & 999.5 & 109.0 & 9.015E-04 & 0.350 & 0.310 & 0.192 & 977.9 & $\begin{array}{l}98.6 \\
\end{array}$ & $1.703 \mathrm{E}-03$ & 0.350 & 0.310 & 0.192 & 1054.1 & 103.7 & $1.346 \mathrm{E}-03$ \\
\hline 0.450 & 0.400 & 0.192 & 999.5 & 108.2 & 9.009E-04 & 0.450 & 0.310 & 0.192 & 977.9 & 97.7 & $1.745 \mathrm{E}-03$ & 0.450 & 0.310 & 0.192 & 1054.1 & 102.4 & $1.397 \mathrm{E}-03$ \\
\hline & & & & & & & & & & & & & & & & & \\
\hline 0.250 & 150 & 225 & 715.2 & 50.4 & $3.662 \mathrm{E}-03$ & 0.250 & 0.150 & 0.225 & 782.0 & 63.5 & 4.103E-03 & 0.250 & 0.150 & 0.225 & 850.8 & 75.0 & 2.611E-03 \\
\hline 0.350 & & & 715.2 & 50.3 & & 0.350 & 0.150 & 0.225 & 782.0 & 62.8 & $4.228 \mathrm{E}-03$ & 0.350 & 0.150 & 0.225 & 850.8 & 73.7 & $2.761 \mathrm{E}-03$ \\
\hline 0.450 & & & 715.2 & 50.3 & & 0.450 & 0.150 & 0.225 & 782.0 & 62.5 & $4.306 \mathrm{E}-03$ & 0.450 & 0.150 & 0.225 & 850.8 & 73.0 & $2.858 \mathrm{E}-03$ \\
\hline 0.250 & 275 & 0.225 & 832.9 & 83.9 & 1.4 & 0.250 & 0.275 & 0.225 & 899.7 & 93.9 & $1.884 \mathrm{E}-03$ & 0.250 & 0.275 & 0.225 & 968.5 & 101.0 & $1.423 \mathrm{E}-03$ \\
\hline 0.350 & 0.275 & 0.225 & 832.9 & 83.4 & $1.423 \mathrm{E}-03$ & 0.350 & 0.275 & 0.225 & 899.7 & 92.3 & $1.946 \mathrm{E}-03$ & 0.350 & 0.275 & 0.225 & 968.5 & 98.7 & $1.498 \mathrm{E}-03$ \\
\hline 0.450 & 0.275 & 0.225 & 832.9 & 83.1 & $1.412 \mathrm{E}-03$ & 0.450 & 0.275 & 0.225 & 899.7 & 91.6 & $1.991 \mathrm{E}-03$ & 0.450 & 0.275 & 0.225 & 968.5 & 97.6 & $1.552 \mathrm{E}-03$ \\
\hline 0.250 & 0.400 & 0.225 & 950.5 & 110.8 & 8.895E-04 & 0.250 & 0.310 & 0.225 & 932.6 & 101.1 & $1.625 \mathrm{E}-03$ & 0.250 & 0.310 & 0.225 & \begin{tabular}{|l|}
1001.4 \\
\end{tabular} & 107.0 & $1.265 \mathrm{E}-03$ \\
\hline 0.350 & 0.400 & 0.225 & 950.5 & 109.6 & $9.009 \mathrm{E}-04$ & 0.350 & 0.310 & 0.225 & 932.6 & \begin{tabular}{|l|}
99.3 \\
\end{tabular} & $1.676 \mathrm{E}-03$ & 0.350 & 0.310 & 0.225 & 1001.4 & 104.4 & $1.327 \mathrm{E}-03$ \\
\hline 0.450 & 0.400 & 0.225 & 950.5 & 109.0 & & 0.450 & 0.310 & 0.225 & 932.6 & 98.5 & $1.714 \mathrm{E}-03$ & 0.450 & 0.310 & 0.225 & 1001.4 & 103.2 & 1.374E-03 \\
\hline & & & & & & & & & & & & & & & & & \\
\hline 0.250 & 0.150 & 0.290 & 696.0 & 50.5 & $3.686 \mathrm{E}-03$ & 0.250 & 0.150 & 0.290 & 756.3 & 63.8 & $3.937 \mathrm{E}-03$ & 0.250 & 0.150 & 0.290 & 818.6 & 75.7 & $2.502 \mathrm{E}-03$ \\
\hline 0.350 & 0.150 & 0.290 & 696.0 & 50.5 & $3.682 \mathrm{E}-03$ & 0.350 & 0.150 & 0.290 & 756.3 & 63.1 & $4.052 \mathrm{E}-03$ & 0.350 & 0.150 & 0.290 & 818.6 & 74.3 & $2.645 \mathrm{E}-03$ \\
\hline 0.450 & 0.150 & 0.290 & 696.0 & 50.4 & $3.696 \mathrm{E}-03$ & 0.450 & 0.150 & 0.290 & 756.3 & 62.8 & 4.125E-03 & 0.450 & 0.150 & 0.290 & 818.6 & 73.7 & $2.738 \mathrm{E}-03$ \\
\hline 0.250 & 0.275 & 0.290 & 797.8 & 84.2 & $1.445 \mathrm{E}-03$ & 0.250 & 0.275 & 0.290 & 858.1 & 94.3 & $1.818 \mathrm{E}-03$ & 0.250 & 0.275 & 0.290 & 920.3 & 101.9 & $1.368 \mathrm{E}-03$ \\
\hline 0.350 & 0.275 & 0.290 & 797.8 & 83.7 & $1.441 \mathrm{E}-03$ & 0.350 & 0.275 & 0.290 & 858.1 & 92.9 & $1.872 \mathrm{E}-03$ & 0.350 & 0.275 & 0.290 & 920.3 & 99.7 & $1.439 \mathrm{E}-03$ \\
\hline 0.450 & 0.275 & 0.290 & 797.8 & 83.4 & $1.429 \mathrm{E}-03$ & 0.450 & 0.275 & 0.290 & 858.1 & 92.1 & $1.912 \mathrm{E}-03$ & 0.450 & 0.275 & 0.290 & 920.3 & 98.6 & $1.490 \mathrm{E}-03$ \\
\hline 0.250 & 0.400 & 0.290 & 899.6 & 111.1 & $9.020 \mathrm{E}-04$ & 0.250 & 0.310 & 0.290 & 886.6 & 101.6 & $1.569 \mathrm{E}-03$ & 0.250 & 0.310 & 0.290 & 948.8 & 108.0 & 1.217E-03 \\
\hline 0.350 & 0.400 & 0.290 & 899.6 & 110.0 & $9.101 \mathrm{E}-04$ & 0.350 & 0.310 & 0.290 & 886.6 & 99.9 & $1.613 \mathrm{E}-03$ & 0.350 & 0.310 & 0.290 & 948.8 & 105.5 & $1.275 \mathrm{E}-03$ \\
\hline 0.450 & 0.400 & 0.290 & 899.6 & 109.4 & $9.075 \mathrm{E}-04$ & 0.450 & 0.310 & 0.290 & 886.6 & 99.1 & 1.647E-03 & 0.450 & 0.310 & 0.290 & 948.8 & 104.3 & 1.319E-03 \\
\hline
\end{tabular}

Figure 18: Results of Back Surface Profile Study

\subsection{Local Reinforcement Study}

Local reinforcement usually refers to localized increases in core web thicknesses. With ULE mirrors in particular, waterjet cutting of the core makes this relatively easy to do. The front and back sheets are usually uniform thickness. There is a mass penalty for this feature, but launch loads and local stresses under pads become a serious issue as the mirrors become lighter and thinner sections. Most of the cases used a fixed reinforcement thickness pattern and pad and perimeter diameters. To see if there was any significant benefit to this local reinforcement, a series of runs were made using the same mesh as reinforced, but keeping all core webs uniform (minimal) thickness (Figure 19). While the previous open back design study indicated that local reinforcement provided a significant benefit, the closed back design does not indicate the same advantage - back plate seems to have eliminated much of the need for local reinforcement.

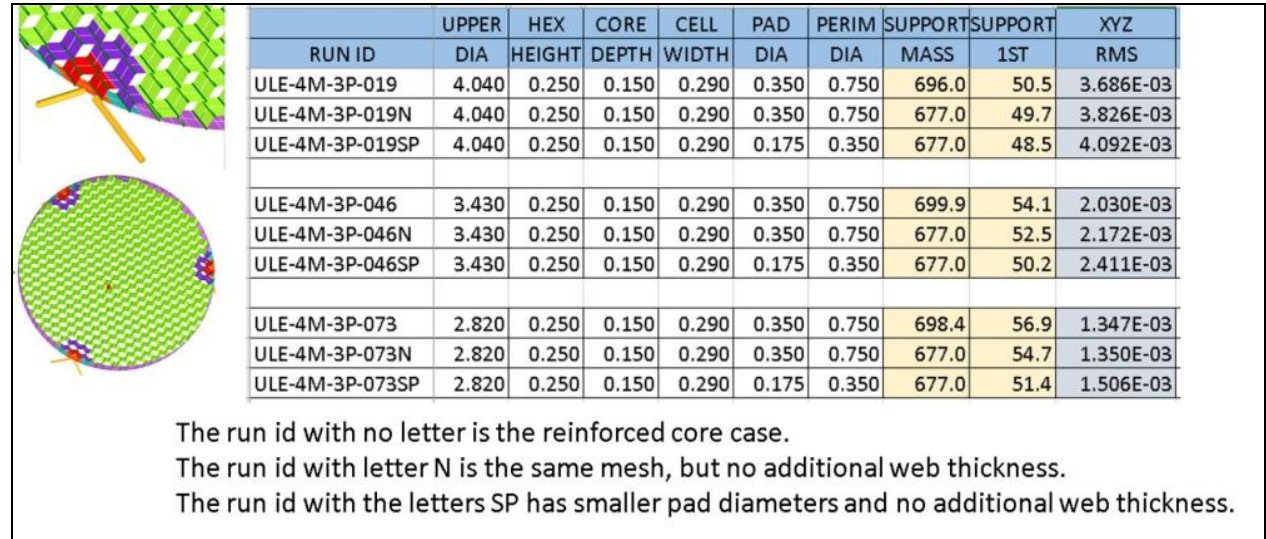

Figure 19: Local Reinforcement Study

SPIE Proceedings 10743, Optical Modeling and Performance Predictions, 2018. 


\subsection{Hexapod Leg Stiffness Study}

All the previous runs used a common strut stiffness value. In order to see if that value was influencing the results, a short parametric study was done using just one geometry case from each mount diameter and 3 and 6 point configurations. The strut stiffness was reduced by two orders of magnitude for each step. The result (Figure 20) showed that once a certain threshold was reached, the actual leg stiffness did not influence the results.

\begin{tabular}{|l|r|r|r|r|r|r|r|r|}
\hline & UPPER & HEX & \multicolumn{1}{|c|}{ LEG } & \multicolumn{1}{c|}{ CORE } & \multicolumn{1}{c|}{ CELL } & SUPPORT & SUPPORT & XYZ \\
\hline RUN ID & DIA & HEIGHT & STIFF & DEPTH & WIDTH & MASS & \multicolumn{1}{c|}{1 ST } & RMS \\
\hline ULE-4M-3P-001 & 4.040 & 0.250 & $8 . E+11$ & 0.150 & 0.192 & 733.7 & 50.2 & $3.626 E-03$ \\
\hline ULE-4M-3P-001A & 4.040 & 0.250 & $8 . E+09$ & 0.150 & 0.192 & 733.7 & 50.1 & $3.628 E-03$ \\
\hline ULE-4M-3P-001B & 4.040 & 0.250 & $8 . E+07$ & 0.150 & 0.192 & 733.7 & 41.69 & $4.469 E-03$ \\
\hline & & & & & & & & \\
\hline ULE-4M-3P-028 & 3.430 & 0.250 & $8 . E+11$ & 0.150 & 0.192 & 741.2 & 54.4 & $1.895 E-03$ \\
\hline ULE-4M-3P-028A & 3.430 & 0.250 & $8 . E+09$ & 0.150 & 0.192 & 741.2 & 54.2 & $1.906 E-03$ \\
\hline ULE-4M-3P-028B & 3.430 & 0.250 & $8 . E+07$ & 0.150 & 0.192 & 741.2 & 35.1 & $8.828 E-03$ \\
\hline & & & & & & & & \\
\hline ULE-4M-3P-055 & 2.820 & 0.250 & $8 . E+11$ & 0.150 & 0.192 & 740.2 & 56.9 & $1.265 E-03$ \\
\hline ULE-4M-3P-055A & 2.820 & 0.250 & $8 . E+09$ & 0.150 & 0.192 & 740.2 & 56.7 & $1.256 E-03$ \\
\hline ULE-4M-3P-055B & 2.820 & 0.250 & $8 . E+07$ & 0.150 & 0.192 & 740.2 & 39.4 & $6.701 E-03$ \\
\hline & & & & & & & \\
\hline ULE-4M-6P-001 & 4.040 & 0.250 & $8 . E+11$ & 0.150 & 0.192 & 759.6 & 33.3 & $1.754 E-02$ \\
\hline ULE-4M-6P-001A & 4.040 & 0.250 & $8 . E+09$ & 0.150 & 0.192 & 759.6 & 33.2 & $1.762 E-02$ \\
\hline ULE-4M-6P-001B & 4.040 & 0.250 & $8 . E+07$ & 0.150 & 0.192 & 759.6 & 26.8 & $2.563 E-02$ \\
\hline & & & & & & & \\
\hline ULE-4M-6P-028 & 3.430 & 0.250 & $8 . E+11$ & 0.150 & 0.192 & 773.9 & 40.4 & $1.094 E-02$ \\
\hline ULE-4M-6P-028A & 3.430 & 0.250 & $8 . E+09$ & 0.150 & 0.192 & 773.9 & 40.3 & $1.103 E-02$ \\
\hline ULE-4M-6P-028B & 3.430 & 0.250 & $8 . E+07$ & 0.150 & 0.192 & 773.9 & 28.2 & $2.047 E-02$ \\
\hline & & & & & & & \\
\hline ULE-4M-6P-055 & 2.820 & 0.250 & $8 . E+11$ & 0.150 & 0.192 & 770.8 & 44.0 & $8.112 E-03$ \\
\hline ULE-4M-6P-055A & 2.820 & 0.250 & $8 . E+09$ & 0.150 & 0.192 & 770.8 & 43.7 & $8.234 E-03$ \\
\hline ULE-4M-6P-055B & 2.820 & 0.250 & $8 . E+07$ & 0.150 & 0.192 & 770.8 & 25.8 & $2.052 E-02$ \\
\hline
\end{tabular}

Figure 20: Hexapod Leg Stiffness Results

\subsection{Facesheet Thickness Study}

Finally, a study of the effect of the facesheet on the mid-frequency Zernikes was done. In the second row of Figure 21, the first number is the thickness of the front-sheet and the second number is the thickness of the back-sheet. Ignoring quilting, no significant improvement is provided by increases up to $13 \mathrm{~mm}$ for either the front only or both plates.

\begin{tabular}{|c|c|c|c|c|c|c|c|c|}
\hline ZERNIKES & \multirow{2}{*}{$\begin{array}{l}\text { WFE Tol } \\
\text { [pm rms] }\end{array}$} & \multicolumn{7}{|c|}{400 Core 190 Cell $100 \%$ Attach Error [pm rms] } \\
\hline K N M & & 1010 & 1110 & 1210 & 1310 & 1111 & 1212 & 1313 \\
\hline 320 Power (Defocus) & 150.00 & 2.335 & 2.335 & 2.341 & 2.354 & 2.354 & 2.379 & 2.407 \\
\hline 422 Pri Astigmatism & 120.00 & 3.033 & 3.137 & 3.241 & 3.345 & 2.949 & 2.880 & 2.822 \\
\hline 531 Pri Coma & 105.00 & 0.047 & 0.047 & 0.046 & 0.046 & 0.047 & 0.047 & 0.048 \\
\hline 640 Pri Spherical & 120.00 & 0.166 & 0.168 & 0.170 & 0.172 & 0.168 & 0.169 & 0.171 \\
\hline 733 Pri Trefoil & 1.56 & 3.094 & 3.094 & 3.102 & 3.116 & 3.115 & 3.142 & 3.175 \\
\hline 842 Sec Astigmatism & 0.21 & 0.144 & 0.149 & 0.154 & 0.159 & 0.141 & 0.138 & 0.135 \\
\hline $951 \mathrm{Sec}$ Coma & 0.21 & 0.009 & 0.009 & 0.010 & 0.011 & 0.009 & 0.010 & 0.010 \\
\hline 1060 Sec Spherical & 0.21 & 0.097 & 0.099 & 0.101 & 0.103 & 0.101 & 0.105 & 0.109 \\
\hline 1144 Pri Tetrafoil & 0.21 & 0.302 & 0.307 & 0.312 & 0.318 & 0.291 & 0.282 & 0.273 \\
\hline $1253 \mathrm{Sec}$ Trefoil & 0.21 & 0.352 & 0.360 & 0.368 & 0.376 & 0.361 & 0.371 & 0.382 \\
\hline 1362 Ter Astigmatism & 0.06 & 0.014 & 0.015 & 0.015 & 0.016 & 0.015 & 0.016 & 0.016 \\
\hline 1471 Ter Coma & 0.06 & 0.011 & 0.011 & 0.012 & 0.012 & 0.011 & 0.012 & 0.012 \\
\hline 1580 Ter Spherical & 0.06 & 0.266 & 0.266 & 0.267 & 0.269 & 0.269 & 0.272 & 0.276 \\
\hline 1655 Pri Pentafoil & 0.21 & 0.342 & 0.354 & 0.365 & 0.376 & 0.342 & 0.342 & 0.342 \\
\hline 1764 Sec Tetrafoil & 0.06 & 0.035 & 0.035 & 0.035 & 0.035 & 0.034 & 0.032 & 0.031 \\
\hline 1873 Ter Trefoil & 0.06 & 0.064 & 0.067 & 0.070 & 0.072 & 0.069 & 0.074 & 0.079 \\
\hline 1982 Qua Astigmatism & 0.06 & 0.007 & 0.007 & 0.007 & 0.007 & 0.007 & 0.006 & 0.006 \\
\hline 2091 Qua Coma & 0.06 & 0.078 & 0.080 & 0.083 & 0.085 & 0.079 & 0.080 & 0.081 \\
\hline 21102 Qua Astigmatism & 0.06 & 0.005 & 0.005 & 0.005 & 0.005 & 0.005 & 0.005 & 0.005 \\
\hline 22120 Qin Spherical & 0.06 & 0.536 & 0.534 & 0.534 & 0.535 & 0.541 & 0.547 & 0.554 \\
\hline
\end{tabular}

Figure 21: Facesheet Thickness Study Results 


\section{CONCLUSIONS}

The Habitable Exoplanet Observatory (HabEx) mission requires a telescope whose optical wavefront has ultra-stable mid-spatial frequency error to enable coronagraphy of exo-Earth class planets. While mass and stiffness are still important design parameters, for coronagraphy, the more important metric is inertial WFE, i.e. the response of the mirror to on-orbit acceleration noise. While an open-back Zerodur mirror is the current baseline, this paper summarizes a series of trade studies for a potential alternative closed-back ULE design. The study looked at over 264 design variations using the Arnold Mirror Modeler and ANSYS@ to investigate the influence of various design elements. As expected, the stiffer the mirror, the better its total rms inertial deformation. But, the distribution of that deformation (i.e. its decomposition into Zernike polynomials) varies with mount configuration (3 vs 6 pads, radial pad location, and strut angle). While previous studies indicated that back shape and local reinforcement were beneficial for open-back mirrors, their benefit was smaller for the closed-back mirrors in this trade study. One conclusion is that the back-sheet eliminates the need for local reinforcement and edge support.

\section{REFERENCES}

[1] Stahl, H. Phillip, "Overview and performance predictions of the baseline 4-meter telescope concept design for habitable-zone exoplanet Observatory",

[2] Arnold, W.R., and Stahl, H. Phillip, "Structural design of a 4-meter off-axis space telescope for the Habitable-zone Exoplanet Direct Imaging Mission", SPIE 10398-7 (2017).

[3] Arnold, W.R., "AMTD design process," NASA/ SPIE Mirror Technology Days, Albuquerque, NM (2014).

[4] Arnold, W.R., Etal, "Next Generation lightweight mirror modeling software", SPIE Opto-mechanical Engineering 2013, San Diego, CA SPIE 8836-15 (2013).

[5] Arnold, W.R., Etal, "Integration of mirror design with suspension system using NASA's new mirror modeling software", SPIE Opto-mechanical Engineering 2013, San Diego, CA SPIE 8836-17 (2013).

[6] Arnold, W.R., Etal, "Next Generation lightweight mirror modeling software", NASA Mirror Tech Days, Redondo Beach, CA (2013). 